\title{
Light-element abundance variations in the Milky Way halo
}

\author{
S. L. Martell and E. K. Grebel
}

\begin{abstract}
Astronomisches Rechen-Institut, Zentrum für Astronomie der Universität Heidelberg, 69120 Heidelberg, Germany
e-mail: martell@ari.uni-heidelberg.de
\end{abstract}

Received 26 January 2010 / Accepted 18 May 2010

\section{ABSTRACT}

\begin{abstract}
We present evidence for the contribution of high-mass globular clusters to the stellar halo of the Galaxy. Using SDSS-II/SEGUE spectra of over $1900 \mathrm{G}$ - and K-type halo giants, we identify for the first time a subset of stars with CN bandstrengths significantly larger, and $\mathrm{CH}$ bandstrengths lower, than the majority of halo field stars, at fixed temperature and metallicity. Since CN bandstrength inhomogeneity and the usual attendant abundance variations are presently understood as a result of star formation in globular clusters, we interpret this subset of halo giants as a result of globular cluster dissolution into the Galactic halo. We find that $2.5 \%$ of our sample is CN-strong, and can infer based on recent models of globular cluster evolution that the fraction of halo field stars initially formed within globular clusters may be as large as $50 \%$.
\end{abstract}

Key words. stars: abundances - Galaxy: halo - galaxies: formation

\section{Introduction}

Hierarchical structure formation is presently the dominant explanation for galaxy formation, based on observed fluctuations in the cosmic microwave background (Hinshaw et al. 2007) and sophisticated numerical simulations of their evolution to the present day (e.g., Diemand et al. 2007; Springel et al. 2008). In this picture, galaxies like the Milky Way are formed through the coalescence of multiple low-mass galaxies which develop within a much larger dark matter halo. The initial disagreement between the calculated mass function of dark matter subhaloes in the simulations and the observed mass function of nearby dwarf galaxies (e.g., Klypin et al. 1999; Moore et al. 1999) is being addressed from several directions at the same time, from complex semianalytic simulations that include star formation and feedback processes, and calculate the chemodynamical evolution of Milky Way-like galaxies (e.g., Johnston et al. 2008; Tumlinson 2010, and references therein) to searches for extremely lowmass galaxies in the Local Group (e.g., Zucker et al. 2006a,b; Belokurov et al. 2007).

The stellar halo of the Milky Way is thought to have been constructed mostly through the early $(\simeq 10$ Gyr ago) accretion of low-mass protogalaxies. The halo exhibits considerable substructure in density and in velocity (e.g. Bell et al. 2008), and kinematically distinct streams presently observed in the halo (e.g., Majewski et al. 2003; Duffau et al. 2006; Martínez-Delgado et al. 2007) are interpreted as remnants of more recent or ongoing merger activity. The "ECHOS" identified in Schlaufman et al. (2009) are interpreted as older substructure that has lost some spatial coherence with time. Studies of the abundance distributions and star formation histories in nearby dwarf galaxies (e.g., Koch et al. 2007a,b, 2008a,b; Kirby et al. 2008; Aoki et al. 2009; Frebel et al. 2009, 2010) have shown that there is a reasonable concordance between the properties of the present-day Milky Way halo (as characterized by, e.g., Schörck et al. 2009) and the dwarf galaxies that would have been available as stellar contributors early in Galactic history (e.g., Font et al. 2006; Carollo et al. 2007).
However, the ongoing dissolution of globular clusters such as Palomar 5 (e.g., Odenkirchen et al. 2001, 2002, 2003; Rockosi et al. 2002; Grillmair \& Dionatos 2006) and NGC 5466 (Belokurov et al. 2006) implies that some fraction of halo stars are initially formed in globular clusters. This dissolution is driven by internal 2-body relaxation, stellar evolution processes and tidal interactions with the Galactic potential, and can cause significant mass loss over the lifetime of typical halo globular clusters (e.g., Gnedin \& Ostriker 1997).

One particular model of globular cluster formation, described in D'Ercole et al. (2008), posits that all old halo clusters surviving to the present day have lost at least $90 \%$ of their initial mass. Early in the development of the cluster, winds from AGB stars collected in the cluster center and formed a second generation of low-mass stars. Type Ia supernovae then caused the cluster to expand, and stars at large radii, mostly members of the first generation, were lost. In the model, the end result, $\simeq 10 \mathrm{Gyr}$ later, is a $10^{5}-10^{6} M_{\odot}$ cluster with two stellar populations that differ slightly in age and abundance pattern.

This two-generation model was developed specifically to explain the anomalous light-element abundance patterns observed in globular cluster stars, specifically the presence in every old globular cluster in the Milky Way of a subpopulation with typical Population II abundances, and a second subgroup with the same metallicity but enhanced $\mathrm{N}, \mathrm{Na}$, and $\mathrm{Mg}$ along with depleted $\mathrm{C}, \mathrm{O}$, and Al. This abundance bimodality has been studied extensively in globular clusters (Langer et al. 1992; Kraft 1994; and Gratton et al. 2004 all provide thorough reviews of the topic), and explanations for the second abundance subgroup have varied from pollution (Cannon et al. 1998) to internal mixing (Langer 1985) to enrichment of star-forming gas by moderate- to high-mass stars (e.g., Cottrell \& DaCosta 1981; Yong et al. 2008) or high-mass binaries (de Mink et al. 2010). Surface pollution of already-formed stars would result in abundance anomalies that are erased at first dredge-up, while current models of deep mixing (e.g., Charbonnel \& Zahn 2007) indicate that it only begins to operate after first dredge-up. Both of these processes have been effectively ruled out as explanations 
for abundance bimodality by the presence of abundance variations at all evolutionary phases in globular clusters (e.g., Briley et al. 2002; Harbeck et al. 2003). In the primordial enrichment scenario, there is ongoing discussion over the exact source of enriching material. In some models the source is moderately high-mass ( 4-5 $M_{\odot}$ ) AGB stars (e.g., Parmentier et al. 1999), while Decressin et al. (2007) claim that rotating high-mass $(M \gtrsim$ $10 M_{\odot}$ ) stars are a better source for processed material because of their very short lifetimes and de Mink et al. (2010) prefer highmass binaries because of their potentially strong mass loss and low wind velocities.

Stars with these light-element abundance anomalies are readily identified through strong $\mathrm{UV} /$ blue $\mathrm{CN}$ molecular absorption and relatively weak absorption in the $\mathrm{CH} \mathrm{G}$ band, and are hereafter called "CN-strong stars", with the understanding that the full abundance pattern from $\mathrm{C}$ through $\mathrm{Al}$ necessarily follows the $\mathrm{CN}$ variation. They are not observed to exist in open clusters (e.g., Smith \& Norris 1984; Jacobson et al. 2008; Martell \& Smith 2009) or the halo field (Gratton et al. 2004). This feedback process apparently only occurs in the high-density environment of globular clusters, and as a result the characteristic sawtooth abundance pattern from carbon through aluminium can be used as a marker of globular cluster origin.

Given the contributions globular clusters are presently making to the halo field, and the significant mass loss predicted theoretically over the lifetime of the Galactic globular cluster system (e.g., Baumgardt et al. 2008), it is intriguing that no CN-strong stars have to date been observed in the halo. We interpret this as a qualitative sign that the contributions to the halo field of globular clusters as we know them today are relatively minor, as is also suggested in Yong et al. (2008).

To test this interpretation, we searched for $\mathrm{CN}$-strong halo giants in the Sloan Extension for Galactic Understanding and Exploration (SEGUE) survey (Yanny et al. 2009). The SEGUE survey is a spectroscopic extension of imaging taken during the Sloan Digital Sky Survey (York et al. 2000), with targets selected to address questions of halo substructure and Galactic formation history. Data were taken from 2005 August through 2008 July using a 640-fiber multiobject spectrograph and the same telescope at Apache Point Observatory that was used for SDSS imaging. The first portion of the SEGUE data was made publically available in 2008 as part of SDSS Data Release 7 (DR7), including roughly 240000 spectra in 200 "pencil beam" lines of sight containing stars chosen for specific purposes (i.e., fields in the Sagittarius stream, M dwarfs to study extremely local kinematic substructure, $\mathrm{G}$ and $\mathrm{K}$ giants to study the distant halo). In addition to flux-calibrated spectra, DR7 also offers the products of the "SEGUE Stellar Parameters Pipeline" (SSPP), which include derived stellar parameters like effective temperature, $[\mathrm{Fe} / \mathrm{H}]$ metallicity, and radial velocity, determined automatically through template matching, $\chi^{2}$ minimization, crosscorrelation or grid-matching methods, as appropriate. Lee et al. (2008a,b) and Allende Prieto et al. (2008) give thorough explanations of the SSPP pipeline and process.

\section{The data set}

Data were obtained from SDSS DR7, through the online Catalog Archive Server. We selected all SEGUE plates, and from those all stars with $[\mathrm{Fe} / \mathrm{H}] \leq-1.0, \log (g) \leq 4.0,(g-r)_{0} \geq 0.2$, and mean signal-to-noise per pixel (SNR) larger than 20 were chosen. Additionally, the errors on various derived parameters were required to be small: $\sigma_{\log (g)} \leq 0.5, \sigma_{[\mathrm{Fe} / \mathrm{H}]} \leq 0.5$ with at least three independent $[\mathrm{Fe} / \mathrm{H}]$ determinations, and reduced $\chi^{2}$ of the

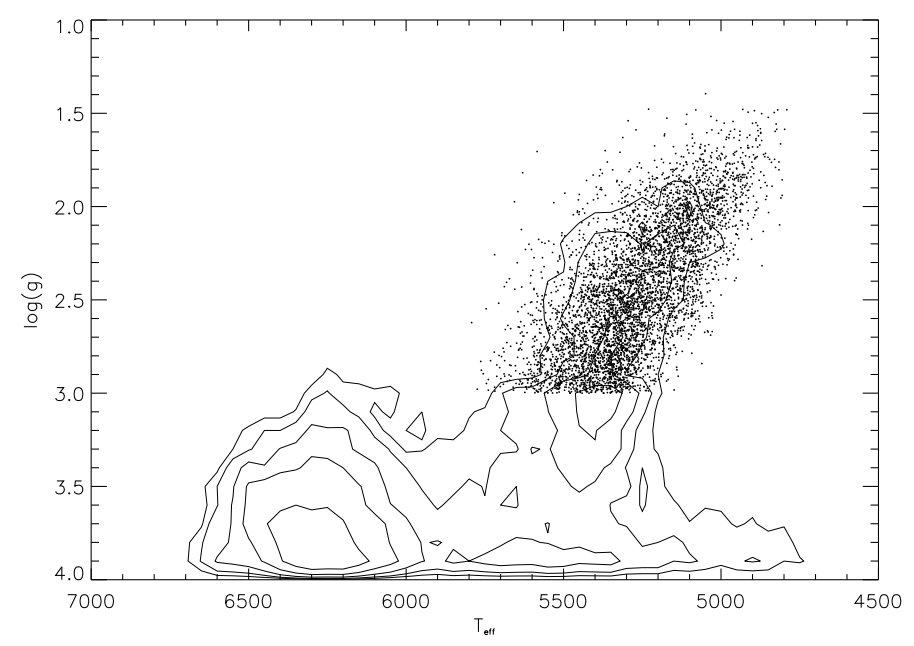

Fig. 1. Hertzsprung-Russell diagram for the initial SEGUE data set, split into two subgroups. $5066 \mathrm{G}$ and $\mathrm{K}$ giants are shown as small points, while density contours for the remaining 17718 stars rejected based on $\left(T_{\text {eff }}, \log (g),[\mathrm{Fe} / \mathrm{H}]\right)$ position are shown as solid lines.

best-fit template spectrum less than 2.0 both in the region of the $\mathrm{Ca}$ II $\mathrm{H}$ and $\mathrm{K}$ lines and in the $\mathrm{CH} \mathrm{G}$ band.

This intentionally generous set of selection criteria then had to be further sub-selected to isolate halo giants. To accomplish this sub-selection, we lowered the limit on $\log (g)$ to 3.0 , then divided the initial data set into 0.2 -dex-wide bins in $[\mathrm{Fe} / \mathrm{H}]$, and removed $\mathrm{AGB}$, main-sequence and turnoff stars from the $\mathrm{H}-\mathrm{R}$ diagram of each of those subsets by rejecting all points more than $3 \sigma$ in $(g-r)_{0}$ from the fiducial sequence. We also made the SNR requirement more stringent, requiring that the mean SNR per pixel in the wavelength range $4000 \leq \lambda \leq 4100$ be larger than 15. This left 5066 halo giants, out of the original 22784 stars. In Fig. 1, the small points represent those halo giants, while density contours of stars rejected based on CMD position are shown as solid lines.

To facilitate later analysis, we convert the dereddened apparent $r_{0}$ magnitudes given in the SEGUE data to absolute $M_{\mathrm{r}}$ magnitudes through a simple photometric parallax calculation. We created a grid of 10 Gyr Padova isochrones (Marigo et al. 2008) with metallicities ranging from $[\mathrm{Fe} / \mathrm{H}]=-1.0$ to $[\mathrm{Fe} / \mathrm{H}]=-1.8$ (the lowest metallicity available) at a spacing of $0.1 \mathrm{dex}$, and interpolate between those to match the metallicity of each individual star. The absolute $M_{\mathrm{r}}$ magnitude corresponding to the observed $(g-r)_{0}$ color of the star, on the interpolated isochrone, is then assigned as the true absolute $M_{\mathrm{r}}$ magnitude.

Errors in $M_{\mathrm{r}}$ were calculated by Monte Carlo sampling of uncorrelated errors in SSPP metallicity, $g_{0}$ magnitude and $r_{0}$ magnitude, drawn randomly from a Gaussian with a width equal to the reported errors on those quantities. This addition of error was done $10^{4}$ times for each star, and we take the standard deviation in those $10^{4}$ determinations of $M_{\mathrm{r}}$ as the error on $M_{\mathrm{r}}$. This error

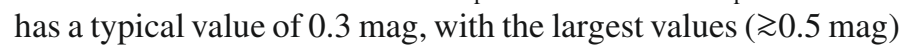
on the $3 \%$ of stars with the largest errors in apparent $r_{0}$ and $g_{0}$. The age of the isochrones used had minimal effects on the derived $M_{\mathrm{r}}$ values, with a change of only $\pm 0.06 \mathrm{mag}$ for a shift of $\mp 1$ Gyr. The limited metallicity range of the isochrones is sufficient for the purposes of the analysis in Sect. 3, but lowermetallicity isochrones would allow us to study the distance distribution of the roughly $1 / 3$ of our final dataset at lower metallicity, to compare the spatial distribution of our final data set to the inner and outer halos identified in Carollo et al. (2007). 


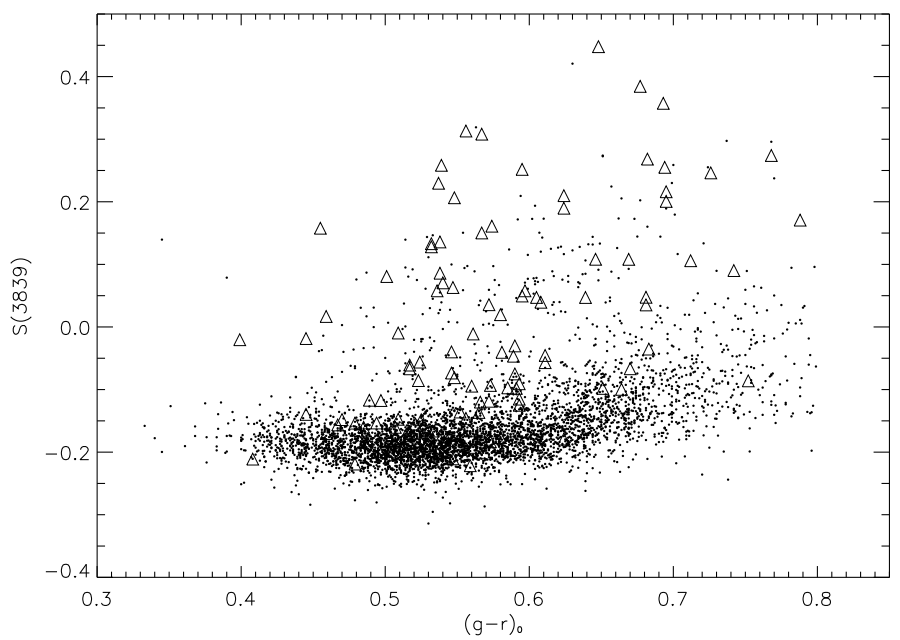

Fig. 2. CN bandstrength index $S$ (3839) versus dereddened $(g-r)$ color for the $\mathrm{G}$ and $\mathrm{K}$ giants indicated in Fig. 1. As expected, the stars are mainly $\mathrm{CN}$-weak, and there is a slight temperature dependence. As mentioned in the text, the stars with dramatically high $S$ (3839) are mainly CEMP and carbon stars (shown as open triangles).

We measured $S$ (3839) (Norris et al. 1981), a bandstrength index for the $\mathrm{CN}$ band at $3883 \AA$, for all halo giant spectra. $S$ (3839) measures the magnitude difference between the integrated flux in the $\mathrm{CN}$ feature and the integrated flux in a nearby continuum band, with more absorption in the feature resulting in larger bandstrength. As can be seen in Fig. 2, there is a strong concentration at low $S(3839)$ in our data set. This is to be expected, since the halo is primarily composed of $\mathrm{CN}$-weak stars with typical Pop. II abundances. There is also a clear trend with temperature, in the sense that cooler stars have larger $\mathrm{CN}$ bandstrengths. The cooling of stars as they ascend the giant branch reddens the spectra and permits more $\mathrm{CN}$ molecule formation, both of which increase the flux difference between the feature and continuum bands of $S$ (3839). There are, however, interesting outliers in Fig. 2: many of the stars with dramatically large $S$ (3839) are carbon stars (shown as open triangles), with correspondingly large $\mathrm{CH}$ and $\mathrm{C}_{2}$ bandstrengths. Figure 3 shows four sample spectra from the halo giant data set: the uppermost spectrum (of SDSS J035123.90+092451.3) is a low-metallicity carbon star, the next (of SDSS J115934.87+002748.0) is a possible CEMP star (Carbon-Enhanced Metal-Poor, having [Fe/H] -2.0 and $[\mathrm{C} / \mathrm{Fe}] \gtrsim+1.0$, and described in Lucatello et al. 2006 and references therein), with strong $\mathrm{CH}$ absorption redward of the $\mathrm{G}$ band and a low metallicity, the next spectrum (of SDSS J064411.96+275351.6) is not a carbon star, but is CN-strong, and the lowest spectrum (of SDSS J145301.24-001954.1) is a typical $\mathrm{CN}$-weak halo star.

The broad molecular features in the example carbon-star spectrum are quite clear. We use the strength of the $\mathrm{CH}$ feature around $4350 \AA$ and the $\operatorname{Swan}(1,0) \mathrm{C}_{2}$ band at $4737 \AA$ to identify carbon and CEMP stars and remove them from the final data set. Specifically, we measure these indices:

$$
\begin{aligned}
& s(c 0)=\frac{\int_{4370}^{4400} f_{\lambda} \mathrm{d} \lambda}{\int_{4330}^{4335} f_{\lambda} \mathrm{d} \lambda+\int_{4440}^{4460} f_{\lambda} \mathrm{d} \lambda} \\
& s(c 1)=\frac{\int_{4660}^{4742} f_{\lambda} \mathrm{d} \lambda}{\int_{4585}^{4620} f_{\lambda} \mathrm{d} \lambda+\int_{4742}^{4800} f_{\lambda} \mathrm{d} \lambda}
\end{aligned}
$$

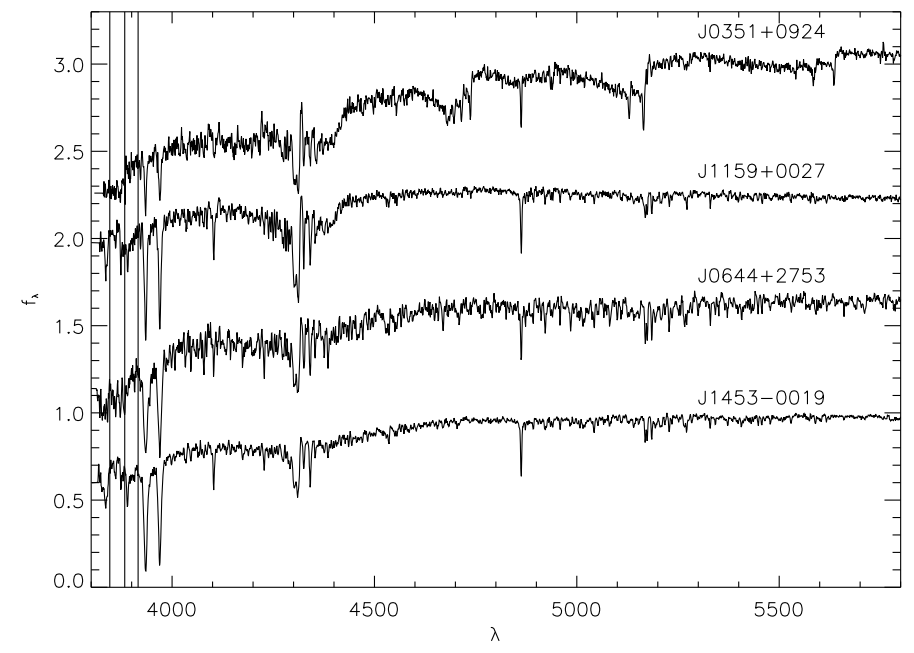

Fig. 3. Sample spectra from the four main types of star comprising our $\mathrm{G}$ and $\mathrm{K}$ giant data set: the topmost is from a carbon star, with clear $\mathrm{CH}$ and $\mathrm{C}_{2}$ bands, and the next is a possible CEMP star, with a metallicity of $[\mathrm{Fe} / \mathrm{H}]=-2.2$ and strong $\mathrm{CH}$ absorption. The next spectrum down is from a CN-strong star, with stronger absorption in the $3883 \AA \mathrm{CN}$ band than the lowest spectrum, which shows the weak UV CN absorption seen in $98 \%$ of our halo giant sample. The vertical lines mark the edges of the $S$ (3839) bands: feature (3846 ̊ to $3883 \AA$ ) and continuum (3883 $\AA$ to $3916 \AA$ ).

and consider all stars with $[\mathrm{Fe} / \mathrm{H}] \leq-1.8$ and $s(c 0) \geq-0.093$; $-1.8 \leq[\mathrm{Fe} / \mathrm{H}] \leq-1.4, s(c 0) \geq-0.05$, and $s(c 1) \geq 0.15$; and $[\mathrm{Fe} / \mathrm{H}] \geq-1.4, s(c 0) \geq-0.02$, and $s(c 1) \geq 0.158$ to have "strong carbon features". Although these carbon and CEMP stars tend to have strong UV CN bands, as is demonstrated in, e.g., Aoki et al. (2002), that is a result of the unusually large ratio of carbon to oxygen in their atmospheres, and is not a result of the anticorrelated $\mathrm{C}-\mathrm{N}$ abundance pattern observed in $\mathrm{CN}$-strong globular cluster stars. There are 109 stars removed from the data set because of strong carbon features, but $\mathrm{CN}$-strong stars are not entirely eliminated: carbon and CEMP stars are shown as open triangles in Fig. 2, and there are clearly other stars with stronger $S(3839)$ than the main group, at fixed $(g-r)_{0}$ color. Of the 109 stars we identify as carbon or CEMP stars, 89 have metallicities below $[\mathrm{Fe} / \mathrm{H}]=-1.8,11$ have metallicities in the range $-1.8 \leq[\mathrm{Fe} / \mathrm{H}] \leq-1.4$, and 9 have a metallicity above $[\mathrm{Fe} / \mathrm{H}]=-1.4$. This is not a surprising metallicity distribution: many studies, including, e.g., Groenewegen (1999), Lucatello et al. (2006) and Aoki et al. (2008), have found that the proportion of carbon stars rises at low metallicity.

\section{Distribution of $\mathrm{CN}$ and $\mathrm{CH}$ bandstrengths}

Since the purpose of this study is identifying stars in the halo with the $\mathrm{CN}$-strong chemical signature associated with globular clusters, and our data source is the moderate-resolution spectroscopy provided by SEGUE, our methods follow closely the techniques used in low-resolution spectroscopic studies of $\mathrm{CN}$ bandstrength behavior in globular clusters. For example, the spectral feature used is the same $3883 \AA \mathrm{CN}$ band, and the index used to measure bandstrength is the well-known $S$ (3839). One first check of this approach is comparing $\mathrm{CN}$ behavior in wellstudied globular clusters to halo stars with similar metallicities observed as part of SEGUE. Figure 4 shows two views of the $\mathrm{CN}$ bandstrength data for M 3, measured from SEGUE spectra of M 3 giants (membership information from J. Smolinski, private 


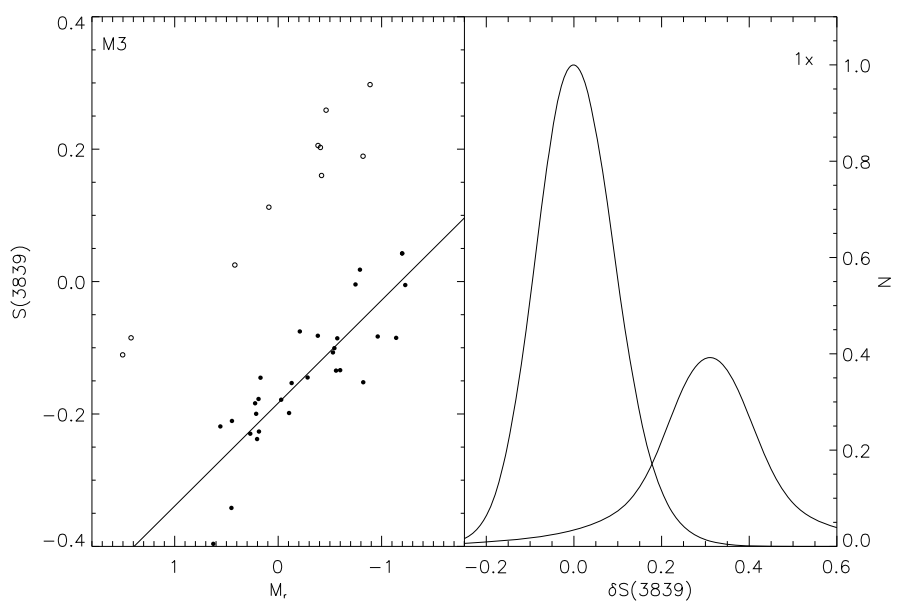

Fig. 4. $S$ (3839) versus $M_{\mathrm{r}}$ and a generalized histogram of $\delta S$ (3839) for M 3 red giants observed by SEGUE. The classic bimodal CN distribution is apparent.

communication). The left panel shows $S$ (3839) versus absolute $M_{\mathrm{r}}$ magnitude, and the typical globular cluster pattern is readily visible: the $S$ (3839) distribution separates into two parallel loci, rising with increasing luminosity. $\mathrm{CN}$-strong stars (shown as open circles) are clearly distinct from $\mathrm{CN}$-weak stars (filled circles) at fixed luminosity.

The right panel of Fig. 4 converts the raw $S$ (3839) data into a generalized histogram. In order to remove the temperaturerelated trend visible in the left panel, we fit a line to the $\mathrm{CN}$-weak locus and measure the quantity $\delta S(3839)$, the vertical difference in $S$ (3839) between every point and the baseline. We then draw a generalized histogram by representing each point as a Gaussian centered at $\delta S$ (3839), with a FWHM equal to the error on $\delta S$ (3839), and summing the individual Gaussians together. In Fig. 4, we base the Gaussian widths in the generalized histogram on the actual measurement errors on $S$ (3839), as determined by Monte Carlo sampling of the spectral error vectors reported by SEGUE. Typical values of $\sigma S$ (3839) are $0.014 \mathrm{mag}$, and we amplify that error by a factor of four in the construction of the generalized histogram. This allows for errors not accounted for in our Monte Carlo noise sampling, particularly errors in flux calibration and the SSPP-derived parameters. We calculate generalized histograms for the $\mathrm{CN}$-strong and $\mathrm{CN}$-weak stars independently, and normalize both curves to the peak of the $\mathrm{CN}$-weak curve, so that the relative heights represent the relative numbers of stars in the two groups.

Figure 5 shows analogous data for field stars in the final data set with $[\mathrm{Fe} / \mathrm{H}]$ within \pm 0.05 dex of $\mathrm{M} 3$, and similar structure can be seen to Fig. 4, with interesting differences in scale. As in Fig. 4, we fit a baseline to the $\mathrm{CN}$-weak stars in the $M_{\mathrm{r}}-S$ (3839) plane (left panel), measure the quantity $\delta S(3839)$, and convert that into a generalized histogram in the right panel. Errors in $\delta S$ (3839) were calculated as in Fig. 4. In this instance, we divide the $\mathrm{CN}$-weak from $\mathrm{CN}$-strong stars by shifting the baseline vertically until it encounters a significant gap. As in Fig. 4, $\mathrm{CN}$-weak stars are shown as filled circles, and CN-strong stars as open circles. Although the two peaks in the right panel have roughly the same separation as in Fig. 4, the $\mathrm{CN}$-weak group is a much more dominant component of the overall population. We have magnified the $\mathrm{CN}$-strong curve in the right panel by a factor of 30 (noted in the upper right corner of the panel) so that it is more easily comparable to the $\mathrm{CN}$-weak curve.

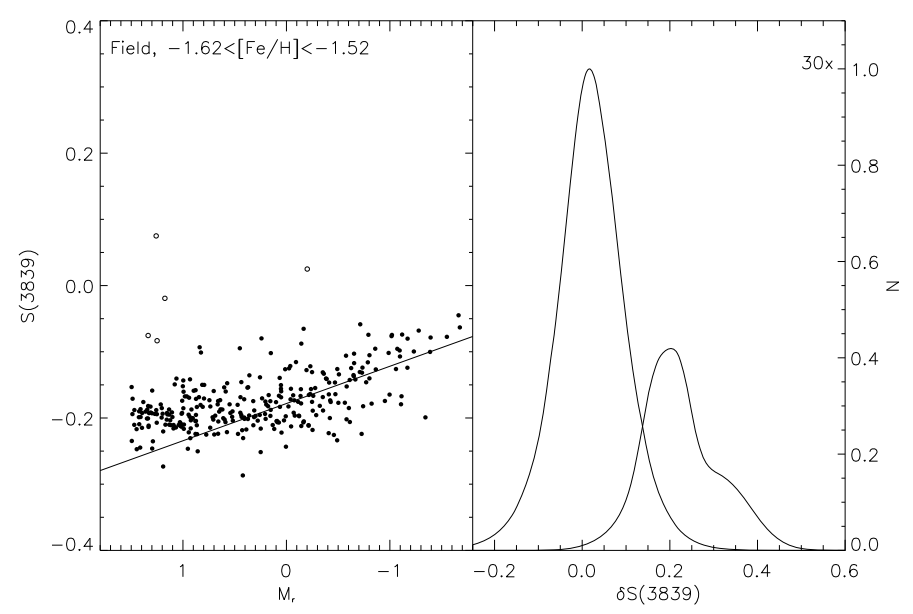

Fig. 5. $S$ (3839) versus $M_{\mathrm{r}}$ and a generalized histogram of $\delta S$ (3839) for field red giants with metallicities similar to $\mathrm{M} 3([\mathrm{Fe} / \mathrm{H}]$ between -1.62 and -1.52). Although $\mathrm{CN}$-weak stars comprise a much larger fraction of the overall data set, there are still relatively $\mathrm{CN}$-strong stars seen. The $\mathrm{CN}$-strong curve has been magnified by a factor of 30 for clarity.

It must be noted that the slope of the baseline in Fig. 5 is different from that in Fig. 4. This is unexpected, since (at fixed metallicity) the same processes ought to be moderating the progressive increase in $\mathrm{CN}$ bandstrength with increasing giantbranch luminosity: a declining temperature that both shifts flux from the science band of $S$ (3839) into the continuum band and permits more $\mathrm{CN}$ molecule formation, along with the "canonical extra mixing" decribed in Denissenkov \& VandenBerg (2003) that progressively depletes carbon and enhances nitrogen abundance in the photosphere. We attribute the difference in baseline slopes to the different mass distributions of the two samples. Specifically, the M 3 giants are an old, single-age population, with masses all around $0.8 M_{\odot}$, while the field giants have the possibility of being considerably younger, and therefore more massive. A younger and more massive red giant, at fixed luminosity, will undergo more rapid evolution, leaving less time for deep mixing and surface abundance changes. Indeed, as is discussed in Gilroy (1989), stars with masses greater than $2.2 M_{\odot}$ evolve along the giant branch too quickly to even begin deep mixing. As a result, younger giants in the field will have weaker $\mathrm{CN}$ bands at a fixed $M_{\mathrm{r}}$, with the effect of pulling down the overall baseline slope.

To extend this type of analysis to the full final data set, we must carefully isolate $[\mathrm{C} / \mathrm{Fe}]$ and $[\mathrm{N} / \mathrm{Fe}]$ variations from the underlying metallicity, which strongly affects the appearance of double-metallic absorption features like $\lambda 3883 \mathrm{CN}$. To that end, we divide the 1958 stars in the final data set into 0.1-dex-wide bins in $[\mathrm{Fe} / \mathrm{H}]$ and search the bins independently for a bimodal distribution of $\mathrm{CN}$ bandstrength at fixed luminosity. Since $\mathrm{CN}$ bandstrength variations become very small at low metallicity, even for significant variations in $[\mathrm{C} / \mathrm{Fe}]$ and $[\mathrm{N} / \mathrm{Fe}]$ (e.g., Martell et al. 2008b; Briley et al. 1993), we limit our sample to the relatively metal-rich end of the halo. Figure 6 shows the raw $S$ (3839) vs. $M_{\mathrm{r}}$ distribution for field stars in each of the metallicity bins between $[\mathrm{Fe} / \mathrm{H}]=-1.0$ and $[\mathrm{Fe} / \mathrm{H}]=-1.8$. The maximum metallicity of the bin is given in the upper left corner of each panel. As in the left panel of Fig. 4, the dashed line in each panel is the baseline against which $\delta S(3839)$ is measured.

There are several features of note in this figure: the slope of the baseline flattens monotonically with dropping metallicity, from -0.11 in the $-1.1 \leq[\mathrm{Fe} / \mathrm{H}] \leq-1.0 \mathrm{bin}$, to -0.04 in 


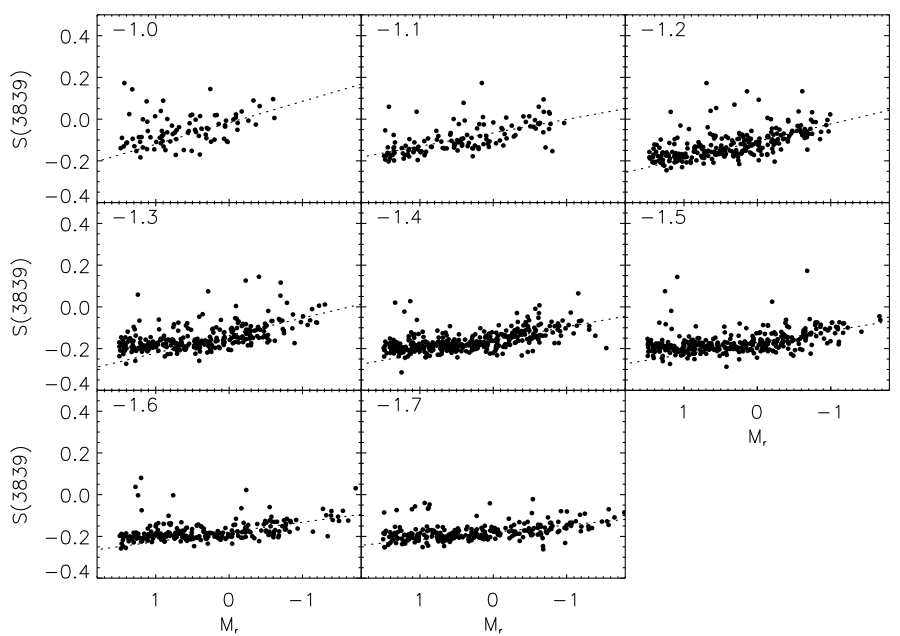

Fig. 6. Raw $S$ (3839) vs. $M_{\mathrm{r}}$ data for the eight 0.1 -dex-wide metallicity bins in our sample. The baselines against which $\delta S(3839)$ is measured are shown as dotted lines, and the maximum metallicity for each panel is given in the upper left corner.

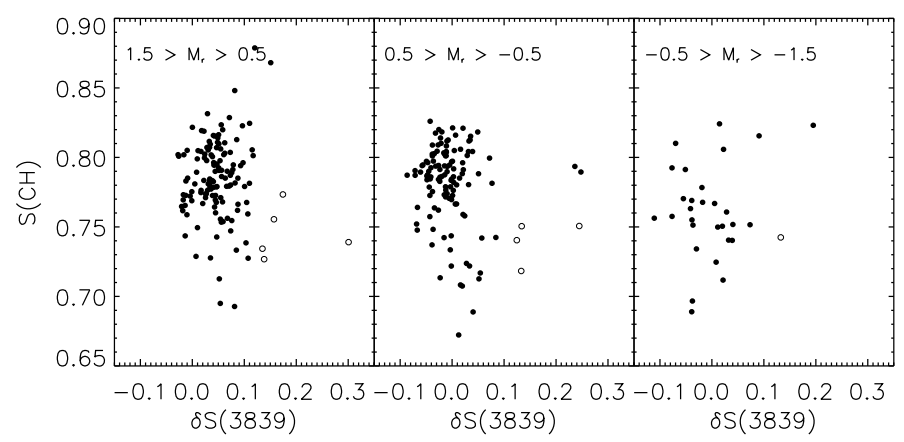

Fig. 7. $\mathrm{CN}$ versus $\mathrm{CH}$ bandstrength for the $-1.4 \leq[\mathrm{Fe} / \mathrm{H}] \leq-1.3$ metallicity bin, divided into three luminosity groups. Stars with both strong $\mathrm{CN}$ and relatively weak $\mathrm{CH}$ are shown as open circles.

the $-1.8 \leq[\mathrm{Fe} / \mathrm{H}] \leq-1.7$ bin. It is known that deep mixing is more efficient at low metallicity: Martell et al. (2008c) find that $\mathrm{d}[\mathrm{C} / \mathrm{Fe}] / \mathrm{dM}_{V}$ is twice as large at $[\mathrm{Fe} / \mathrm{H}]=-2.3$ as at -1.0 , and Sweigart \& Mengel (1979) predict less-compressed hydrogenburning shells, allowing for more penetration by meridional circulation currents, at lower $[\mathrm{Fe} / \mathrm{H}]$. In addition, the gap between the $\mathrm{CN}$-strong and $\mathrm{CN}$-normal groups shrinks as metallicity declines, an effect that occurs even without reducing the size of variations in $[\mathrm{C} / \mathrm{Fe}]$ and $[\mathrm{N} / \mathrm{Fe}]$.

In order to identify stars with globular cluster-like carbon and nitrogen abundances in Fig. 6, we look for the classic $\mathrm{CN}-\mathrm{CH}$ anticorrelation, used in globular cluster studies as a clear signal of carbon depletion and nitrogen enrichment. Figure 7 shows $\delta S(3839)$ versus the $\mathrm{CH}$ bandstrength index $S(\mathrm{CH})$ (Martell et al. 2008a) for the subset of the final data set with $-1.4 \leq[\mathrm{Fe} / \mathrm{H}] \leq-1.3$. The stars are further subdivided by absolute $M_{\mathrm{r}}$ magnitude, since $\mathrm{CH}$ bandstrength is a sensitive function of both temperature and carbon abundance, and both decrease with rising luminosity. Stars with relatively large $\delta S$ (3839) and relatively weak $S(\mathrm{CH})$ in each luminosity sub-bin are shown as open circles.

We repeated this selection process, dividing the metallicity bins into luminosity sub-bins, for the other seven metallicity bins, and Fig. 8 shows $\delta S(3839)$ versus $S(\mathrm{CH})$ for each of the metallicity bins, with all luminosity sub-bins collapsed together. Stars with strong $\mathrm{CN}$ (and weak $\mathrm{CH}$, in the lower-metallicity

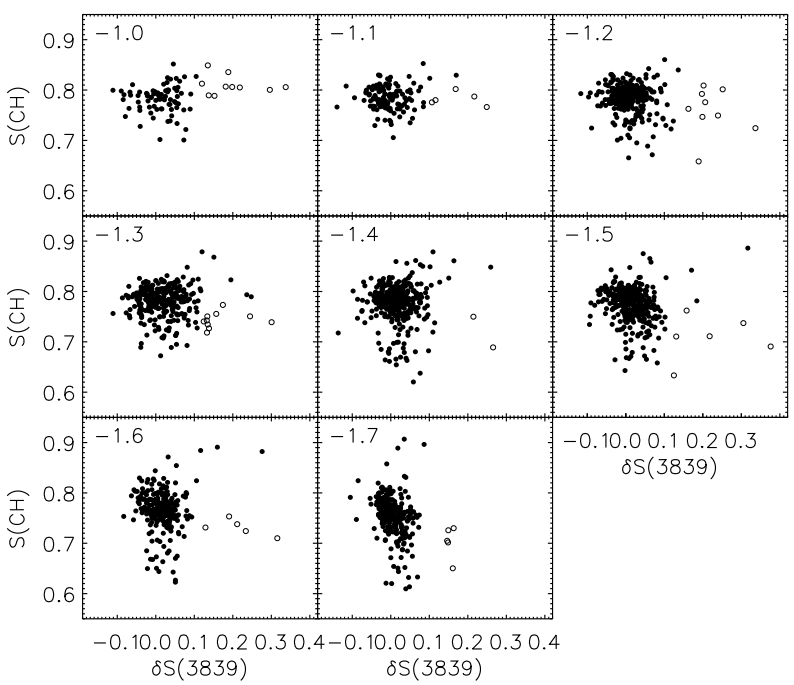

Fig. 8. $\mathrm{CN}$ versus $\mathrm{CH}$ bandstrength for all eight metallicity bins, selected from luminosity sub-bins as in Fig. 7. Candidate CN-strong stars are shown as open circles.

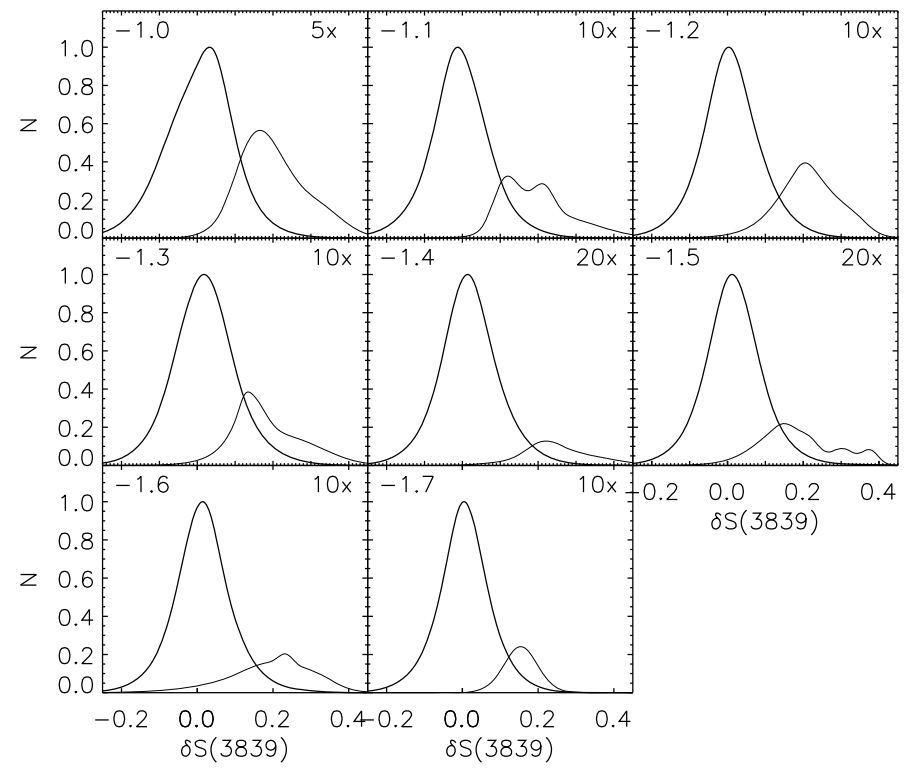

Fig. 9. Generalized histograms of $\delta S(3839)$ for $\mathrm{CN}$-weak and $\mathrm{CN}$-strong stars in each $[\mathrm{Fe} / \mathrm{H}]$ bin. As in Fig. 6, maximum metallicity for each panel is given in the upper left corner. As in Fig. 5, CN-strong histograms were amplified for clarity. The multiplicative factor for each panel is given in the upper right corner.

bins), relative to the majority of halo field stars, are shown as open circles in each panel. Altogether, we identify 49 stars (also shown as open circles in Fig. 8) as relatively $\mathrm{CN}$-strong and $\mathrm{CH}$-weak. Our $\mathrm{CH}$ bandstrength index $S(\mathrm{CH})$ is calibrated based on bright red giants in the low-metallicity globular cluster M 53. It is therefore less responsive to variations in carbon abundance in high-metallicity stars and in fainter giants than it was designed for. However, it was the most responsive over the full parameter range of our data, of the nine G-band indices we considered. As a result, we are less stringent in our $\mathrm{CH}$ bandstrength selection in the higher-metallicity bins than in the lower-metallicity bins.

The eight panels of Fig. 9 correspond to the panels in Fig. 6, and show generalized histograms of $\delta S$ (3839) for the $\mathrm{CN}$-normal and $\mathrm{CN}$-strong stars in each metallicity bin, using the $\mathrm{CN}$ bandstrength classifications made in Fig. 8. As in Fig. 5, 
the CN-strong curves needed to be amplified to be easily visible next to the $\mathrm{CN}$-weak curves; the multiplicative factor is given in the upper right corner of each panel, and the maximum metallicity in each bin is given in the upper left corner of each panel. These generalized histograms are qualitatively similar to Fig. 4, although with a clearly different $\mathrm{CN}$-strong/CN-weak ratio: there is a separation between the two peaks of roughly 0.2 mag in $\delta S(3839)$, which shrinks as the overall metallicity drops.

The presence of stars in the halo field with relatively strong $\lambda 3883 \mathrm{CN}$ absorption and relatively weak absorption in the $\lambda 4320 \mathrm{CH}$ G band, at fixed metallicity and luminosity, is a strong indication that globular clusters have contributed stars to the halo field. Given current models for the origin of light-element abundance variations in globular clusters, it does not seem possible that these stars formed in the halo with these atypical $[\mathrm{C} / \mathrm{Fe}]$ and $[\mathrm{N} / \mathrm{Fe}]$ abundances. An investigation of the abundances of $\mathrm{O}, \mathrm{Na}$, $\mathrm{Mg}$ and $\mathrm{Al}$, which are also known to vary in $\mathrm{CN}$-strong globular cluster stars (see, e.g., Kraft 1994), would allow a more strict test, and possibly a more firm confirmation, of our claim that the 49 halo field stars we identify here as $\mathrm{CN}$-strong originated in globular clusters.

For completeness, we also mention the possibility that these stars did not originate within globular clusters, but rather that their unusual abundances are the result of mass transfer from an AGB companion in a binary system. However, as is demonstrated in Lucatello et al. (2005), which discusses binarity in CEMP stars, the fraction of field stars expected to be in a binary system with a companion of 3-8 $M_{\odot}$ and orbital parameters that permit evolution of the companion up to the AGB phase, and then mass transfer but not coalescence, is quite small. There is no high-precision radial velocity monitoring program in progress for these stars; we predict that such a program would be unlikely to find binary companions.

\section{Discussion}

Although light-element abundance variations have not been observed before in the halo, our identification of these $\mathrm{CN}$-strong halo field stars is not wholly unexpected. There are several wellunderstood mechanisms for globular cluster mass loss, and theoretical studies of globular cluster formation and evolution (e.g., D'Ercole et al. 2008; Baumgardt et al. 2008) predict significant mass loss in individual clusters as well as a dramatic reshaping of the cluster mass function with time. The data set we analyzed, selected from the SEGUE survey, is not representative of the full halo, in mass, evolutionary phase, or metallicity. Distances to the candidate $\mathrm{CN}$-strong stars range from 4 to nearly $40 \mathrm{kpc}$, with $93 \%$ found within $20 \mathrm{kpc}$ of the Sun. However, our result for red giants is generalizable to all halo stars, since abundance bimodality is observed to exist at all masses and evolutionary phases in globular clusters. More fundamentally, all of the cuts we made in selecting the final data set are blind to $\mathrm{CN}$ and $\mathrm{CH}$ bandstrengths and light-element abundances, and all nitrogenenhanced giants with metallicities above $[\mathrm{Fe} / \mathrm{H}]=-1.8$ ought to show clearly strong $\mathrm{CN}$ bands. Since approximately $2.5 \%$ of our halo red giants exhibit strong $\mathrm{CN}$ bands and weak $\mathrm{CH}$ bands, we expect that the same fraction of the entire halo will contain the same abundance enhancements and depletions. This prediction can be confirmed by observations of dwarfs in the halo field, because main sequence stars in globular clusters show the same abundance division as giants (e.g., Briley et al. 2002).

In order to convert $f_{\mathrm{h}}^{\mathrm{p}}$, the present-day fraction of $\mathrm{CN}$-strong halo stars, into $f_{\mathrm{h}}^{\mathrm{gc}}$, the fraction of globular cluster-originating stars in the halo field, we must consider what fraction $\mathrm{CN}$-strong stars comprise of the stars originally formed in globular clusters. In the two-generation scenario of D'Ercole et al. (2008), roughly $90 \%$ of stars originally formed in a globular cluster, consisting entirely of first-generation stars (with halo-like chemistry), are lost between the epoch of cluster formation and the present day. This means that $f_{\mathrm{gc}}^{\mathrm{m}}$, the fraction of stars that remain as members of the globular cluster they were formed in, is around 0.1 . Since $f_{\mathrm{gc}}^{\mathrm{p}}$, the fraction of present-day globular clusters stars that are CN-strong, is around 0.5 (e.g., Kraft 1994), we can calculate that $f_{\mathrm{h}}^{\mathrm{gc}}=\frac{f_{\mathrm{h}}^{\mathrm{p}}}{f_{\mathrm{gc}}^{\mathrm{m}} \times f_{\mathrm{gc}}^{\mathrm{p}}}$. Since $f_{\mathrm{h}}^{\mathrm{p}}=0.025$ in the present study, this suggests that $f_{\mathrm{h}}^{\mathrm{gc}}=\frac{0.025}{0.1 \times 0.5}=0.5$, and that a remarkable $50 \%$ of the halo field originally formed in the massive star clusters that were progenitors of the present-day globular cluster population, with a further unknown contribution of $\mathrm{CN}$-weak stars made by globular clusters that did not survive to the present day and were not massive enough to self-enrich.

While some numerical studies of galaxy formation (e.g., Boley et al. 2009) have suggested that the halo could be constructed entirely from disrupted globular clusters, there is not presently a strong consensus on the role of globular clusters in cosmological-scale galaxy formation. Precise numerical study of the dynamical evolution of globular clusters is very complicated: the number of particles is large enough, and the relevant timescales short enough, that highly accurate simulations are very time-consuming. However, the development of semianalytic prescriptions for the mass evolution of globular clusters would allow single-halo-scale simulations like those of Johnston et al. (2008) to include them as a source of halo stars, and to predict what fraction of the halo field ought to originate in globular clusters.

Acknowledgements. S.L.M. wishes to thank Graeme Smith and Tim Beers for helpful conversations about this project. Funding for the SDSS and SDSSII has been provided by the Alfred P. Sloan Foundation, the Participating Institutions, the National Science Foundation, the US Department of Energy, the National Aeronautics and Space Administration, the Japanese Monbukagakusho, the Max Planck Society, and the Higher Education Funding Council for England. The SDSS Web Site is http://www.sdss.org/. The SDSS is managed by the Astrophysical Research Consortium for the Participating Institutions. The Participating Institutions are the American Museum of Natural History, Astrophysical Institute Potsdam, University of Basel, University of Cambridge, Case Western Reserve University, University of Chicago, Drexel University, Fermilab, the Institute for Advanced Study, the Japan Participation Group, Johns Hopkins University, the Joint Institute for Nuclear Astrophysics, the Kavli Institute for Particle Astrophysics and Cosmology, the Korean Scientist Group, the Chinese Academy of Sciences (LAMOST), Los Alamos National Laboratory, the Max-Planck-Institute for Astronomy (MPIA), the Max-Planck-Institute for Astrophysics (MPA), New Mexico State University, Ohio State University, University of Pittsburgh, University of Portsmouth, Princeton University, the United States Naval Observatory, and the University of Washington.

\section{References}

Allende Prieto, C., Sivarani, T., Beers, T. C., et al. 2008, AJ, 136, 2070 Aoki, W., Norris, J. E., Ryan, S. G., Beers, T. C., \& Ando, H. 2002, ApJ, 567, 1166

Aoki, W., Beers, T. C., Sivarani, T., et al. 2008, ApJ, 678, 1351

Aoki, W., Arimoto, N., Sadakane, K., et al. 2009, A\&A, 502, 569

Baumgardt, H., Kroupa, P., \& Parmentier, G. 2008, MNRAS, 384, 1231

Bell, E. F., Zucker, D. B., Belokurov, V., et al. 2008, ApJ, 680, 295

Belokurov, V., Evans, N. W., Irwin, M. J., Hewett, P. C., \& Wilkinson, M. I.

2006, ApJ, 637, L29

Belokurov, V., Zucker, D. B., Evans, N. W., et al. 2007, ApJ, 654, 897

Boley, A. C., Lake, G., Read, J., \& Teyssier, R. 2009, ApJ, 706, L192

Briley, M. M., Cohen, J. G., \& Stetson, P. B. 2002, ApJ, 579, L17

Briley, M. M., Smith, G. H., Hesser, J. E., \& Bell, R. A. 1993, AJ, 106, 142 
Cannon, R. D., Croke, B. F. W., Bell, R. A., Hesser, J. E., \& Stathakis, R. A 1998, MNRAS, 298, 601

Carollo, D., Beers, T. C., Lee, Y. S., et al. 2007, Nature, 450, 1020

Charbonnel, C., \& Zahn, J.-P. 2007, A\&A, 467, L15

Cottrell, P. L., \& Da Costa, G. S. 1981, ApJ, 245, L79

de Mink, S. E., Pols, O. R., Langer, N., \& Izzard, R. G. 2010, IAU Symp., 266, 169

Decressin, T., Charbonnel, C., \& Meynet, G. 2007, A\&A, 475, 859

Denissenkov, P. A., \& VandenBerg, D. A. 2003, ApJ, 593, 509

D'Ercole, A., Vesperini, E., D’Antona, F., McMillan, S. L. W., \& Recchi, S. 2008, MNRAS, 391, 825

Diemand, J., Kuhlen, M., \& Madau, P. 2007, ApJ, 667, 859

Duffau, S., Zinn, R., Vivas, A. K., et al. 2006, ApJ, 636, L97

Font, A. S., Johnston, K. V., Bullock, J. S., \& Robertson, B. E. 2006, ApJ, 638, 585

Frebel, A., Kirby, E., \& Simon, J. D. 2009, Nature, 464, 72

Frebel, A., Simon, J. D., Geha, M., \& Willman, B. 2010, ApJ, 708, 560

Gilroy, K. K. 1989, ApJ, 347, 835

Gnedin, O. Y., \& Ostriker, J. P. 1997, ApJ, 474, 223

Gratton, R., Sneden, C., \& Carretta, E. 2004, ARA\&A, 42, 385

Grillmair, C. J., \& Dionatos, O. 2006, ApJ, 641, L37

Groenewegen, M. A. T. 1999, in Asymptotic Giant Branch Stars, ed. T.

Le Bertre, A. Lebre, \& C. Waelkens, IAU Symp., 191, 535

Harbeck, D., Smith, G. H., \& Grebel, E. K. 2003, AJ, 125, 197

Hinshaw, G., Nolta, M. R., Bennett, C. L., et al. 2007, Ap\&SS, 170, 288

Jacobson, H. R., Friel, E. D., \& Pilachowski, C. A. 2008, AJ, 135, 2341

Johnston, K. V., Bullock, J. S., Sharma, S., et al. 2008, ApJ, 689, 936

Kirby, E. N., Simon, J. D., Geha, M., Guhathakurta, P., \& Frebel, A. 2008, ApJ, $685, \mathrm{~L} 43$

Klypin, A., Kravtsov, A. V., Valenzuela, O., \& Prada, F. 1999, ApJ, 522, 82

Koch, A., Grebel, E. K., Kleyna, J. T., et al. 2007a, AJ, 133, 270

Koch, A., Wilkinson, M. I., Kleyna, J. T., et al. 2007b, ApJ, 657, 241

Koch, A., Grebel, E. K., Gilmore, G. F., et al. 2008a, AJ, 135, 1580

Koch, A., McWilliam, A., Grebel, E. K., Zucker, D. B., \& Belokurov, V. 2008b, ApJ, 688, L13
Kraft, R. P. 1994, PASP, 106, 553

Langer, G. E. 1985, PASP, 97, 382

Langer, G. E., Suntzeff, N. B., \& Kraft, R. P. 1992, PASP, 104, 523

Lee, Y. S., Beers, T. C., Sivarani, T., et al. 2008a, AJ, 136, 2022

Lee, Y. S., Beers, T. C., Sivarani, T., et al. 2008b, AJ, 136, 2050

Lucatello, S., Tsangarides, S., Beers, T. C., et al. 2005, ApJ, 625, 825

Lucatello, S., Beers, T. C., Christlieb, N., et al. 2006, ApJ, 652, L37

Majewski, S. R., Skrutskie, M. F., Weinberg, M. D., \& Ostheimer, J. C. 2003, ApJ, 599, 1082

Marigo, P., Girardi, L., Bressan, A., et al. 2008, A\&A, 482, 883

Martell, S. L., \& Smith, G. H. 2009, PASP, 121, 577

Martell, S. L., Smith, G. H., \& Briley, M. M. 2008a, PASP, 120, 839

Martell, S. L., Smith, G. H., \& Briley, M. M. 2008b, PASP, 120, 7

Martell, S. L., Smith, G. H., \& Briley, M. M. 2008c, AJ, 136, 2522

Martínez-Delgado, D., Peñarrubia, J., Jurić, M., Alfaro, E. J., \& Ivezić, Z. 2007, ApJ, 660, 1264

Moore, B., Ghigna, S., Governato, F., et al. 1999, ApJ, 524, L19

Norris, J., Cottrell, P. L., Freeman, K. C., \& Da Costa, G. S. 1981, ApJ, 244, 205

Odenkirchen, M., Grebel, E. K., Dehnen, W., Rix, H., \& Cudworth, K. M. 2002 , AJ, 124, 1497

Odenkirchen, M., Grebel, E. K., Rockosi, C. M., et al. 2001, ApJ, 548, L165

Odenkirchen, M., Grebel, E. K., Dehnen, W., et al. 2003, AJ, 126, 2385

Parmentier, G., Jehin, E., Magain, P., et al. 1999, A\&A, 352, 138

Rockosi, C. M., Odenkirchen, M., Grebel, E. K., et al. 2002, AJ, 124, 349

Schlaufman, K. C., Rockosi, C. M., Beers, T. C., et al. 2009, ApJ, 703, 2177

Schörck, T., Christlieb, N., Cohen, J. G., et al. 2009, A\&A, 507, 817

Smith, G. H., \& Norris, J. 1984, AJ, 89, 263

Springel, V., Wang, J., Vogelsberger, M., et al. 2008, MNRAS, 391, 1685

Sweigart, A. V., \& Mengel, J. G. 1979, ApJ, 229, 624

Tumlinson, J. 2010, ApJ, 708, 1398

Yanny, B., Rockosi, C., Newberg, H. J., et al. 2009, AJ, 137, 4377

Yong, D., Meléndez, J., Cunha, K., et al. 2008, ApJ, 689, 1020

York, D. G., Adelman, J., Anderson, Jr., J. E., et al. 2000, AJ, 120, 1579

Zucker, D. B., Belokurov, V., Evans, N. W., et al. 2006a, ApJ, 650, L41

Zucker, D. B., Belokurov, V., Evans, N. W., et al. 2006b, ApJ, 643, L103 